\title{
Symposium: 2014 Annual Meeting of the Limb Lengthening and Reconstruction Society
}

\section{Editorial Comment: 2014 Annual Meeting of the Limb Lengthening and Reconstruction Society}

\author{
Sanjeev Sabharwal MD, MPH
}

\footnotetext{
I
}

$t$ is often debated whether certain medical specialty organizations, such as the Limb Lengthening and Reconstruction Society (LLRS), are too narrow in their clinical focus to thrive and engage a large audience. Others feel that the problem may be excessive overlap with orthopedic specialties, such as adult reconstruction, trauma, pediatrics, and foot and ankle.

The author certifies that he, or any members of his immediate family, has no commercial associations (eg, consultancies, stock ownership, equity interest, patent/licensing arrangements, etc) that might pose a conflict of interest in connection with the submitted article.

All ICMJE Conflict of Interest Forms for authors and Clinical Orthopaedics and Related Research ${ }^{\mathbb{R}}$ editors and board members are on file with the publication and can be viewed on request.

The opinions expressed are those of the writers, and do not reflect the opinion or policy of $\operatorname{CORR}^{\circledR}$ or The Association of Bone and Joint Surgeons ${ }^{\mathbb{R}}$.

S. Sabharwal MD, MPH ( $\square)$

Department of Orthopedics, Rutgers -

New Jersey Medical School, 90 Bergen

Street, Suite 7300, Newark,

NJ 07101-1709, USA

e-mail: sabharsa@njms.rutgers.edu
I think this is more of an issue of perception than reality; in fact, this little society brings big ideas together, and rather than overlapping with those other specialty areas, the LLRS connects them-and so the work of the LLRS should be of interest to many, including many surgeons who are not LLRS members. While lengthening limbs is a small part of what most members of LLRS do, the cross-pollination amongst various subspecialties brings the multinational members together, fostering a growth mindset and generating new ideas for addressing old problems related to limb deformities. Topics like osteomyelitis, reconstruction for various congenital and acquired deformities, including those following trauma are all are within the purview of the LLRS, but are important elements of the practices of many surgeons outside it. It is for this reason that we partner with Clinical Orthopaedics and Related Research $^{\mathbb{R}}$ - to get selected papers presented at our meetings in front of as many readers beyond our small society as we can.

Even the more narrowly construed topics that come up at LLRS (like limb deformity) should be of interest in

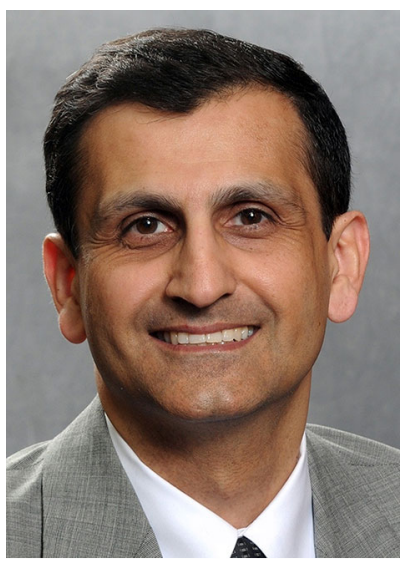

Sanjeev sabharwal MD, MPH

mainstream orthopaedics since they raise key principles that apply to practices of many different disciplines within the specialty. Is it not true that we all assess the patient presenting with an angular limb deformity prior to recommending treatment? Is it not imperative for the surgeon to identify the origin (bone and/or soft tissue), plane, and magnitude of each deformity, irrespective of the patient's age and underlying etiology, before a customized treatment plan is executed? Surgeons who fail to do so, risk creating iatrogenic deformities. Furthermore, with evolving technology, 


\section{Symposium: 2014 Annual Meeting of the Limb Lengthening and Reconstruction Society}

such as lengthening nails, software-assisted external fixators, and fixatorassisted fixation, there are more userfriendly ways to reconstruct limbs. It is important that the principles raised in articles evaluating the safety and efficacy of such new technologies are relevant to our clinical practice. While these advan- ces can often streamline the surgical experience, there is a lurking temptation to try these newer devices before gaining a deeper understanding into the biology of distraction osteogenesis and fully appreciating the unique pitfalls of each implant. We must not yield to this temptation; designers should be held to a high stan- dard of study, and nondesigner validation studies should be performed before a new tool is adopted broadly.

That said, I hope you enjoy the following papers, culled from our 2014 annual meeting. To find out more about LLRS activities, please visit our website at www.1lrs.org. 